Article

\title{
Parity-Time Symmetry and the Toy Models of Gain-Loss Dynamics near the Real Kato's Exceptional Points
}

\author{
Miloslav Znojil \\ Nuclear Physics Institute of CAS, Hlavni 130, 25068 Řež, Czech Republic; znojil@ujf.cas.cz; \\ Tel.: +420-266-173-286 \\ Academic Editor: Blas Manuel Rodríguez-Lara \\ Received: 16 May 2016; Accepted: 12 June 2016; Published: 20 June 2016
}

\begin{abstract}
For a given operator $D(t)$ of an observable in theoretical parity-time symmetric quantum physics (or for its evolution-generator analogues in the experimental gain-loss classical optics, etc.) the instant $t_{\text {critical }}$ of a spontaneous breakdown of the parity-time alias gain-loss symmetry should be given, in the rigorous language of mathematics, the Kato's name of an "exceptional point", $t_{\text {critical }}=t^{(E P)}$. In the majority of conventional applications the exceptional point $(E P)$ values are not real. In our paper, we pay attention to several exactly tractable toy-model evolutions for which at least some of the values of $t^{(E P)}$ become real. These values are interpreted as "instants of a catastrophe", be it classical or quantum. In the classical optical setting the discrete nature of our toy models might make them amenable to simulations. In the latter context the instant of Big Bang is mentioned as an illustrative sample of possible physical meaning of such an EP catastrophe in quantum cosmology.
\end{abstract}

Keywords: parity-time symmetry; Schrödinger equation; physical Hilbert space; inner-product metric operator; real exceptional points; solvable models; quantum Big Bang; quantum Inflation period

\section{Introduction}

In a historical perspective, the recent enormous growth of interest in the experimental as well as theoretical aspects of optical systems exhibiting various anomalous (typically, complex and space-dependent) forms of refraction index $\mathfrak{n}(\vec{x}) \notin \mathbb{R}$ (mostly in two dimensions, with $\vec{x} \in \mathbb{R}^{2}$ ) may be traced back to the influential theoretical letter [1] in which Carl Bender with his student Stefan Boettcher recalled the traditional parity-time symmetry alias $\mathcal{P} \mathcal{T}$ symmetry and transferred its use from relativistic quantum field physics (e.g., [2]) to the Krein-space-related spectral-theory mathematics [3,4]. In this manner Bender with Boettcher opened the Pandora's box of possible applications of the concept of $\mathcal{P} \mathcal{T}$ symmetry

$$
H \mathcal{P} \mathcal{T}=\mathcal{P} \mathcal{T} H
$$

of Hamiltonians $H$ in non-relativistic quantum mechanics [5]. In parallel, their study inspired also generalizations of mathematically similar theories in several other branches of physics including even the classical mechanics and, first of all, classical optics (also [6]; updated historical and contextual remarks and also many related fresh as well as older references may be found, e.g., in the physics-reviewing chapter [7] and/or in the more mathematics-oriented rest of monograph [8]).

The combination of the $\mathcal{P} \mathcal{T}$-symmetric quantum theory with the predominantly experimental nature of the study of gain-loss systems in classical optics proved unexpectedly fortunate and productive. In the past, the collaboration between the two communities of researchers materialized, 
i.a., during the series of dedicated international conferences [9]. There also seem to be no reasons for a decrease of intensity of this mutual inspiration in the future. In what follows, the latter optimistic expectation is to be supported constructively, via a toy-model study of the systems and instants at which, in the language of quantum physicists, the $\mathcal{P} \mathcal{T}$-symmetry becomes spontaneously broken.

We intend to present here several new ideas in the field, with emphasis upon their possible experimental testability in classical optics as well as upon their potentially deep impact during the future development of quantum theory and of its various less standard phenomenological applications.

\section{The Context of Quantum Mechanics}

Our basic methodical idea is that it makes sense to develop further the recently revealed intimate formal relationship between the theoretically appealing quantum theories exhibiting $\mathcal{P} \mathcal{T}$-symmetry (typically, in the Schrödinger equation) and the experimentally realizable classical optical systems built, basically, on the solutions of the formally similar Maxwell equations in paraxial approximation. Briefly, all of these systems may be characterized by the various specific forms of the balance between the sinks and sources, causing the gain and loss in the electromagnetic radiation in general and in the intensity of the light in optical settings in particular.

On the less pragmatic level of theoretical speculations we would like to point out here that in both the classical and quantum contexts of propagation dynamics the most interesting phenomena may be expected to occur, in the language of optics, in the domains of a "deeply complex" refraction. In the parallel language of abstract quantum theory, one should speak about the anomalously-looking dynamical regime of an apparently "deeply non-unitary" evolution, i.e., with reference to the famous Stone's theorem [10], about the regime in which the quantum Hamiltonian appears "deeply non-self-adjoint".

Once we recall the unifying terminology of mathematics we reveal that a common background of study may be found in the classical Kato's book on linear operators [11]. The author explained there the necessary mathematical concepts via a methodically extremely efficient replacement of complicated differential generators of evolution by the mere $N$-dimensional matrices $D^{(N)}$, mostly of the first nontrivial dimension $N=2$. In this manner, Kato was able to circumvent virtually all of the inessential technical complications which mar, typically, the wider acceptance of the mathematically very complicated differential-operator quantum Hamiltonians of Bender and Boettcher [12]. At the same time, the use of the matrix models with $N<\infty$ may be also expected to facilitate the building of bridges between the simplified matrix forms of toy-model $\mathcal{P} \mathcal{T}$-symmetric quantum Hamiltonians $H=D^{(N)}$ and their optical analogs which would be much more accessible to the experimental testing.

The above-mentioned ambiguous concepts of the "deeply complex" refraction and/or of the "deeply non-self-adjoint" operators of observables lose immediately their misleading flavor after one recalls the Kato's terminology. Indeed, one immediately imagines that the qualitative features of the generic evolution law

$$
\mathrm{i} \partial_{t}|\psi\rangle=H|\psi\rangle
$$

(i.e., of quantum Schrödinger equation or of its formally equivalent paraxial-approximation analogue in classical optics) are really best understood when the complicated differential operators $H$ (i.e., typically, the Bender's and Boettcher's $\mathcal{P} \mathcal{T}$-symmetric Hamiltonians $H=-d^{2} / d x^{2}+V(x)$ with complex potentials $V(x)$ such that $H \mathcal{P} \mathcal{T}=\mathcal{P} \mathcal{T} H$ [1]) are approximated or entirely replaced by the suitable toy-model matrices $D^{(N)}$ for which $\mathcal{T}$ performs Hermitian conjugation while the parity-simulating $\mathcal{P}$ may be virtually arbitrary invertible matrix, exhibiting usually just the involution-representation property $\mathcal{P}^{2}=I$.

A particularly friendly class of time-dependent (and, incidentally, real) $N$ by $N$ matrices $D^{(N)}(t)$ representing a quantum observable (i.e., admissibly but not necessarily just the evolution operators $H$ entering Equation (2)) was constructed and studied in [13]. Speaking, for the sake of brevity, just the language of quantum theory from now on, the concept of the "deeply non-self-adjoint" dynamical 
regime was given there an entirely rigorous mathematical meaning. Moreover, the very first element of the family

$$
D^{(2)}(t)=\left[\begin{array}{cc}
-1 & \sqrt{1-t^{2}} \\
-\sqrt{1-t^{2}} & 1
\end{array}\right], \quad t \in(-\infty, \infty)
$$

(where $t$ may denote either time or another variable parameter) was chosen equivalent to one of the Kato's illustrative examples.

Due to the latter incidental coincidence of toy models we imagined, later, that the physics-inspired "deeply non-self-adjoint" operators are strictly those in which the parameter (i.e., in our model (3), time t) lies sufficiently close to its so called "exceptional point" value $t^{(E P)}$ at which, by definition [11], the matrix in question acquires a non-diagonalizable, Jordan-block-equivalent form. For our most elementary toy model (3) there exists strictly one EP value which is real, $t^{(E P)}=0$ [14].

After the toy-model specification of $H=D^{(2)}(t)$, the "physical" solutions of Schrödinger Equation (2) would cease to be acceptable at the EP value of time. This immediately leads to the interesting questions of what can happen and what happens under which circumstances (i.e., what is the physical meaning and dynamics of the system) in the EP vicinity, at the small and positive differences $t-t^{(E P)}$. The latter problem will be addressed in what follows.

\section{The Context of the Theory of Catastrophes}

\subsection{A Remark on Terminology}

Long before the ultimate formulation of the $\mathcal{P} \mathcal{T}$-symmetric quantum theory between years 2002 and 2004 (cf., e.g., its most compact summary in [6]) many EP-related paradoxes found already comparatively straightforward clarifications in the context of physics [15] as well as in the context of mathematics [16]. The message provided by the recent return ad fontes is that what is usually called an unbroken $\mathcal{P} \mathcal{T}$-symmetry dynamical regime in physics should have better been called a quasi-Hermitian dynamical regime.

Let us add that the latter, much less confusing term was proposed, in the framework of nuclear physics, in the older and, alas, a much less known review paper [17]. After the attention of international community returned to this review and after the applications of the quasi-Hermitian representations of observables were transferred to the other branches of physics, the above-mentioned family of toy models $D^{(N)}(t)$ sampled by Equation (3) has been extended, in $[13,18,19]$, to all matrix dimensions $N<\infty$. Subsequently, an interesting phenomenological application of the model was proposed in [20]. Matrices $D^{(N)}(t)$ were reinterpreted there as Hamiltonians $H=H_{0}+V$ of the usual perturbation-theory form containing a - presumably small - variable component $V$. The key physical message as delivered by [20] was a warning that due to the manifest non-Hermiticity of $H \neq H^{\dagger}$ in the conventional, "first" Hilbert space $\mathbb{R}^{N}=\mathcal{H}^{(F)}$ the impact of a small change of $V$ may happen to be abrupt and non-perturbative.

The latter result was interpreted as a certain quantum analogue of the classical Thom's catastrophic scenario called "cusp" [21,22]. The argument proceeded via a reinterpretation of perturbation $V$, the strength of which was not measured by $t$ but rather by a small coupling constant $\lambda$ such that $t= \pm \sqrt{\lambda}$. Such a change of perspective implied that at the small negative couplings $\lambda<0$ the spectra of energies became purely imaginary and, hence, unphysical. The EP values $\lambda_{(E P)}=0$ themselves acquired, at any dimension $N$, the status of the points of an abrupt loss of stability alias of the points of a quantum catastrophe. Needless to add that precisely such a loss of stability seems amenable to the experimental simulations also in the framework of classical optics (also [23]).

\subsection{The Dyson's Maps in Quantum Theory}

The main mathematical result of [20] was that before an onset of the catastrophe, i.e., under the real-energy constraint $\lambda=\lambda(t)>0$ the evolution controlled by the toy-model Hamiltonians 
$H=D^{(N)}(t)$ may be kept unitary, provided only that one replaces the above-selected false Hilbert space $\mathcal{H}^{(F)}=\mathbb{R}^{N}$ (in which $H$ was $\mathcal{P} \mathcal{T}$-symmetric but non-Hermitian) by its "second", standard physical alternative $\mathcal{H}^{(S)}$ in which $H$ would be selfadjoint. In the manner recalling the older idea of Dyson [15] the goal has been achieved by the ad hoc amendment of the Hermitian-conjugation operation,

$$
\mathcal{T}^{(S)}: H \rightarrow H^{\ddagger}=\Theta^{-1} H^{\dagger} \Theta
$$

Although, as we already indicated, the trick itself was already well known in mathematics $[8,16]$ as well as in physics [5-7,17], the key innovation was that the operator $\Theta$ playing the role of an inner-product metric has been shown obtainable, in closed form, for all of the tridiagonal toy-model matrices $D^{(N)}(t)$ of the family [20].

The EP-possessing toy-model Hamiltonians $H(\lambda)=D^{(N)}(t)$ with non-negative couplings were mathematically well defined and self-adjoint in the reconstructed physical Hilbert space $\mathcal{H}^{(S)}$. The accompanying exact, closed-form knowledge of the necessary operator $\Theta$ yielding $H=H^{\ddagger}$ allowed one to perform all of the calculations in the unphysical but certainly friendlier auxiliary Hilbert space $\mathcal{H}^{(F)}$.

In principle (though hardly in practice) the reconstructed physical Hilbert space $\mathcal{H}^{(S)}$ may be also replaced by its unitarily equivalent "third space" partner $\mathcal{H}^{(T)}$. [17,24,25] may be consulted for all of the technical details of the latter equivalence transformation. For our present purposes it suffices to mention that the mapping $\Omega$ which connects the latter two vector spaces $\mathcal{H}^{(S)}$ and $\mathcal{H}^{(T)}$ also appears in definition $\Theta=\Omega^{\dagger} \Omega$ of the Hilbert-space metric.

In $\mathcal{H}^{(T)}$ the role of the isospectral partner of a generic observable $D^{(N)}(t)$ is played by operator $\mathfrak{d}^{(N)}(t)$ such that

$$
D^{(N)}(t)=\Omega^{-1}(t) \mathfrak{d}^{(N)}(t) \Omega(t), \quad \Omega^{\dagger}(t) \Omega(t)=\Theta(t) \neq I
$$

In the traditional applications of the formalism, operator $\mathfrak{d}^{(N)}(t)$ is always more complicated and much less user-friendly than its partner even in the simplified, finite-dimensional models. Otherwise, people feel that one would not have had any reason for changing the representation.

\subsection{Special Models with Real Exceptional Points}

In a mathematically oriented monograph [11] Kato studied certain parameter-dependent families of operators $\hat{D}(t)$ or, in a simplified setting, of $N$ by $N$ matrices $D^{(N)}(t)$ in the framework of perturbation theory. He introduced the formal concept of the so called exceptional point $t_{(E P)}$ as such (in general, complex) value of parameter $t$ at which, roughly speaking, there emerges the so called Jordan block structure in the canonical form of $\hat{D}(t)$.

In the monograph, the introduction of the concept was illustrated by several two-by-two examples. Thus, one can pick up matrix (3) with two real $t$-dependent eigenvalues $d_{ \pm}(t)= \pm t$. In this example it is easy to see that the two eigenvectors as well as the two eigenvalues are well separated at $t \neq 0$ but coincide at $t=t_{(E P)}=0$. In other words, the EP matrix $D^{(2)}(0)$ ceases to be diagonalizable. It becomes merely unitarily equivalent to a non-diagonal Jordan-block matrix,

$$
-\frac{1}{2} D^{(2)}(0) \sim\left[\begin{array}{ll}
0 & 1 \\
0 & 0
\end{array}\right]
$$

In the same mathematical context of perturbation theory the exact solvability and the real-EP property (5) of the $N=2$ toy model was also shown to hold, in [13], for certain less trivial three-by-three toy-model matrices, for the sequence of four-by-four matrices as well as for the whole family of subsequent $N$ by $N$ tridiagonal-matrix descendants $D^{(N)}(t)$ of these models at any higher matrix dimension $N$ (also [18]). 


\section{The Context of Classical Optics}

\subsection{Solvable Quantum Models}

By the, up to now, more or less purely formal change of representation (4), the main problem of the phenomenological acceptability of the family of quantum toy models of [13] becomes transferred from the description of the spectrum of $D^{(N)}(t)$ (which has been shown trivial in [19]) to the construction of the mapping $\Omega(t)$ (which is, usually, enormously difficult in practice [7]). In this sense, a decisive merit of working with toy models of [13] may be seen in the feasibility of such a construction, i.e., of the construction of the related metric $\Theta(t)$ and/or of the mappings $\Omega(t)$ themselves.

In applications, the knowledge of the latter operators could immediately lead, in principle at least, not only to the backward reconstruction $D^{(N)}(t) \rightarrow \mathfrak{d}^{(N)}(t)$ but also to the analogues of the mapping (4) for multiple other observables (including the Hamiltonian), the operator representations of which are usually much better known in $\mathcal{H}^{(T)}$, mainly due to the principle of correspondence [26].

\subsection{Paraxial Approximation and Anomalous Diffusion Phenomena}

During the early discussions of the concept of the parity times time-reversal symmetry alias $\mathcal{P} \mathcal{T}$-symmetry in 2001 it was, among others, Alexandr Turbiner who expressed his opinion that in the context of quantum theory there must exist a certain form of equivalence between its Hermitian and $\mathcal{P} \mathcal{T}$-symmetric representations [27]. Later on, his intuitive expectations have been confirmed in constructive manner (cf., e.g., [6]).

At the same time, paradoxically, what also survived, until now, was the present author's reply, counterargument and prediction saying, basically, that from the point of view of phenomenology the crucial differences between the textbook and $\mathcal{P} \mathcal{T}$-symmetric quantum theories will emerge in the dynamical regime in which the $\mathcal{P} \mathcal{T}$-symmetry gets lost during a new form of a phase transition (cf., e.g., [28]). In the context of abstract quantum theory we already mentioned above, that and how one of the productive confirmations of the latter prediction emerged, later on, in the context of the quantum theory of catastrophes in which the Kato's purely mathematical concept of exceptional points was put in direct contact with the phenomenologically crucial instant of the spontaneous breakdown of $\mathcal{P} \mathcal{T}$-symmetry.

Unexpectedly enough, several years later, the latter prediction of the relevance of EPs also found an independent and, perhaps, even more persuasive confirmation in the context of classical optics (for details see, in particular, a historically oriented Section 1.7.3 of the multi-authored monograph [8]). In this setting, serendipitously, the correspondence was established via a discovery [29-31] of the strict mathematical equivalence between certain quantum Schrödinger equations and their classical Maxwell-equation partners in their so called paraxial-approximation versions which describe the classical optical diffraction.

In spite of the apparent marginality of the latter classical-quantum correspondence, its consequences proved extremely important, for several reasons. The first reason was that in the quantum context the generic non-Hermiticity of Hamiltonians and/or of the further operators of observable quantities made the immediate experimental verifications or even tests of the theory and of its various toy models extremely difficult. In contrast, the facilitated feasibility of the analogous experiments in classical optics profited from an enormous speed of progress in contemporary nanotechnologies and, in particular, in the recently opening possibilities of manufacture of various synthetic materials exhibiting precisely the required $\mathcal{P} \mathcal{T}$-symmetry-guaranteeing properties of the necessary anomalous optical systems.

The second comparative advantage of performing experiments in classical optics reflected the mathematical complexity of the analogous quantum models in which only the accessibility of the "realistic" Dyson's maps $\Omega$ and/or of their probability-determining metric-operator products (4) enables one to quantify all of the required experimental predictions [17]. 
There exist several other arguments which act in favor of working with the classical-optics representations. On the level of mathematics it is encouraging to see that, in optics, many interesting, $\mathcal{P} \mathcal{T}$-symmetry-related phenomena are already observable via the most elementary, discrete, finite-element toy models [29,31]. Secondly, on the level of phenomenology let us recall numerous conceptual and/or technical difficulties emerging in the $\mathcal{P} \mathcal{T}$-symmetric models of the standard unitary quantum scattering [32]. The complexity of these obstacles is in a huge contrast with the simplicity of the experimental realizations of multiple simulations of various analogous dispersive phenomena in classical optics (one can even take there certain nonlinearities into account [33]).

\section{The Context of Quantum Cosmology}

We have to admit that the most extreme contrast between the expected feasibility of an experimental optical simulation and an absolute inaccessibility of any experimental study in an analogous quantum setting may and should be sought in the framework of quantum cosmology. The existence of such a challenge has already been noticed in a conference contribution [34]. Its mathematically highly challenging nature was emphasized there, together with its deep relevance for several characteristic quantum cosmology scenarios. At the same time we were only able to work there, non-numerically, with the two by two matrix observables. Still we believe that before a transfer of the quantum-evolution problems from the speculative cosmology to the experimental domain of classical optics one should succeed in moving from the two by two toy-model matrices to the higher matrix dimensions $N=3, \ldots$

\subsection{Problems with Quantization}

In the recent literature devoted to cosmology the singularities predicted by classical theory are widely believed to be smeared out after quantization. In particular, the spatial degeneracy at Big Bang is often replaced by its Big-Bounce alternative $[35,36]$. We propose that such a strong belief should be weakened. Constructively, we intend to show that the survival of the singularities sampled by Big Bang could find one of its possible explanations in the exceptional-point (EP, [11]) interpretation of the Big-Bang time $t=0$.

We shall describe an elementary, very schematic family of non-covariant toy-model realization of such a possibility. We will work with a schematic, empty Universe which is quantized in a non-covariant version of Heisenberg picture [37]. In such a setting the classical Big Bang singularity may survive the quantization.

In the model the mechanism of the unfolding of the singularity after Big Bang will be shown to carry certain, not fully clarified features including, e.g., a strong initial anisotropy of Hilbert space which weakens and isotropizes in finite time. We believe that such a result could offer also a hint or nonstandard key to the existence and/or quantum-theoretical origin of the Inflation period in the evolution of the Universe.

\subsection{The Consistent Quantum Singularity at Big Bang}

Let us now return to the question of the most interesting physical interpretations of operator $D^{(N)}(t)$. Basically, the answer lies in the possibility of treating this operator as the distance between two observers in an empty space (Universe). In a cosmology-oriented Gedankenexperiment, such a pair of observers (let us call them, say, Alice and Bob-they may be perceived as attached, say, to different Galaxies) are assumed to measure their (classical) distance $d=d(t)$ (cf. Figure 1). Once they extrapolate their data to remote past, they may reveal the existence of the Big Bang singularity,

$$
\lim _{t \rightarrow t_{c}=0} d(t)=0
$$




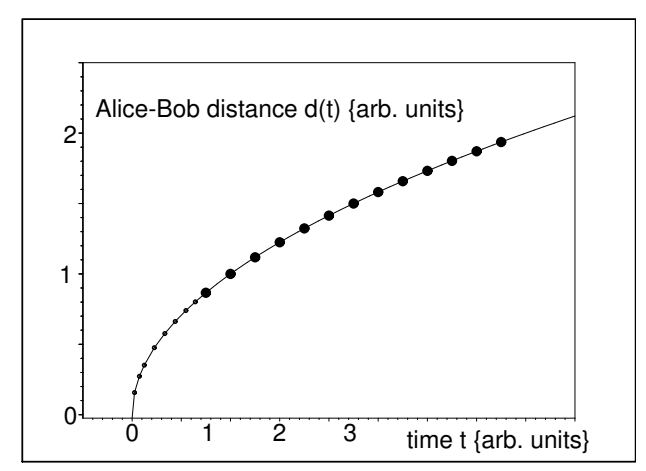

Figure 1. The Alice-Bob distances $d(t)$ : (1) measured by the methods of classical physics (large dots), (2) measured, in a Gedankenexperiment, by quantum-theory methods (including, perhaps, also the hypothetical "superluminal-velocity" Inflation period, small dots), (3) extrapolated down to $t=0$ (smooth curve, quantum Big-Bang-singularity hypothesis).

Hypothetically, Alice and Bob may have started their experiment very early after the Big Bang. In such a case one should recall the principle of classical-quantum correspondence and replace the real curve $d(t)$ by the pencil $\mathfrak{d}(t)$ of self-adjoint operators defined in the above-mentioned alternative physical Hilbert space $\mathcal{H}^{(T)}$. In Heisenberg picture (and, say, in pure state), the wave function $|\psi\rangle$ of the Alice's and Bob's Universe will be time-independent while, under adiabatic hypothesis, their measured distance will smoothly continue the classical curve and, moreover, it will coincide, in the case of a luckily chosen quantum theory, with one of the eigenvalues of the toy-model operator $\mathfrak{d}(t)$.

A decisive weakness of the whole argument has already been noticed by Ashtekar et al. [35,38,39]. The essence of the problem may be seen in the incompatibility of the quantized version of the degeneracy of eigenvalues (6) with the self-adjoint nature of the underlying operator. Indeed, from the purely mathematical point of view the eigenvalues of $\mathfrak{d}\left(t_{c}\right)$ may degenerate either at a complex value of the EP Big Bang time $t_{c}$ or after an emergence of some mysterious ad hoc symmetry at some real value of $t_{c}$. Both of these possibilities look, clearly, unacceptable.

In the manner which was already proposed in the not too successful illustrative construction [34], the escape out of such an apparent no-go theorem may be provided by the time-dependent generalization (cf. [24,40]) of the Dyson's [15] non-unitary isospectral change of representation. As long as we realize all of our constructions solely in the auxiliary Hilbert space $\mathcal{H}^{(F)}$, the mapping (4) leads to the manifestly non-Hermitian operator representation $D^{(N)}(t)$ of the observable Alice-Bob distance. Hence, there are no formal objections against the critical EP time of the Big Bang being real (and, for simplicity, shifted to the origin, $t=t_{c}=0$ ).

\section{Toy Models}

In the language of mathematics, the problem we intend to discuss here is the problem of the behavior of a quantum/classical system near its multiple, degenerate exceptional-point singularity. We believe that a particularly suitable class of models suitable for such a study are the discrete, $N$ by $N$ matrix models. Their purely formally motivated but remarkably transparent class was proposed in Refs. $[13,18,19]$. In what follows, the low-dimensional toy models of this class will be explored as most suitable candidates for bridging the existing gaps between the formulation of certain quantum-cosmological hypotheses and some future experimental optical simulations and tests of structures and stability of their descriptive properties.

\subsection{Toy Model Matrices with $N=2$}

For times $t>1$ (i.e., long after the passage of the system through its exceptional point-in a way inspired by cosmology [41,42] let us call this passage, briefly, "Big Bang") our $N=2$ toy-model 
operator (3) (representing any generic observable quantity-in the light of our forthcoming, phenomenology-oriented applications of the formalism let us call this observable, for the sake of brevity, "distance") is self-adjoint even in the friendly Hilbert space $\mathcal{H}^{(F)}=\mathbb{R}^{2}$. Due to the extreme simplicity of such an illustrative model we may recall constraint $\mathfrak{d}=\mathfrak{d}^{\dagger}\left(\right.$ in $\mathcal{H}^{(T)}=\mathbb{R}^{2}$ ) alias

$$
D^{\dagger}(t) \Theta(t)=\Theta(t) D(t), \quad t \neq 0
$$

(in $\mathcal{H}^{(F)}=\mathbb{R}^{2}$ ) and construct all of the Hermitizing inner-product metrics $\Theta(t)$, i.e., all of the eligible, manifestly time-dependent physical Hilbert spaces $\mathcal{H}^{(S)}[43,44]$. The exhaustive, complete menu of our Hilbert space metrics (HSMs) at $N=2$,

$$
\Theta=\Theta_{[\alpha]}^{(2)}(t)=\left[\begin{array}{cc}
1+t \sin \alpha & -\sqrt{1-t^{2}} \\
-\sqrt{1-t^{2}} & 1-t \sin \alpha
\end{array}\right]
$$

is defined in terms of a single [25] free real parameter $\alpha$ such that $-\pi / 2<\alpha<\pi / 2$. Both of the eigenvalues of the metric are positive,

$$
\theta_{ \pm}=1 \pm \sqrt{1-t^{2} \cos ^{2} \alpha}>0
$$

At any time $t \neq t_{B B}=0$ each choice of $\alpha$ specifies a different inner product, i.e., a different, potentially physical Hilbert space $\mathcal{H}^{(S)}=\mathcal{H}_{[\alpha]}^{(S)}(t)$. This means that the physical contents of the model described by operator (3) and by the related $\operatorname{HSM} \Theta_{[\alpha]}(t)$ will vary with $\alpha$.

One of the most successful strategies of removal of such an unpleasant ambiguity was proposed by Bender et al. [45]. They recommended the use of ansatz $\Theta=\mathcal{P C}$ where $\mathcal{P}$ (in the diagonal matrix form where $\mathcal{P}_{n n}=(-1)^{n}$ ) mimics parity and where $\mathcal{C}$ (such that $\mathcal{C}^{2}=e^{2} I$ ) mimics charge. In our case this conjecture leads to formula

$$
\mathcal{C}_{[\alpha]}^{(2)}(t)=\left[\begin{array}{cc}
1+t \sin \alpha & -\sqrt{1-t^{2}} \\
\sqrt{1-t^{2}} & -1+t \sin \alpha
\end{array}\right]
$$

and to constraint $\alpha=\alpha_{\text {optimal }}=0$, with several remarkable consequences. Firstly, the choice of $\alpha_{\text {optimal }}$ minimizes the anisotropy $\delta_{[\alpha]}^{(2)}=\left|\theta_{+}-\theta_{-}\right|=2 \sqrt{1-t^{2} \cos ^{2} \alpha}$ of the Hilbert-space geometry at all $t \in(0,1)$. Secondly, as long as we have $\delta_{\text {optimal }}^{(2)}=\delta_{[0]}^{(2)}=2 \sqrt{1-t^{2}}$, the anisotropy will vanish yielding the trivial, isotropic-metric limit $\Theta_{\text {optimal }}(t)=I$ at $t=1$.

As long as the smooth continuation of Equation (3) to the larger times $t>1$ would make our only observable $D^{(2)}(t)$ manifestly Hermitian, the related physical HSM matrix could be kept trivial as well, $\Theta_{\text {optimal }}(t)=I$ at $t>1$. In this case the interval of times in which the HSM matrix $\Theta_{\text {optimal }}(t) \neq I$ is anomalous will be finite, yielding $\mathcal{H}^{(S)}(t)=\mathcal{H}^{(F)}$ at all $t \in(1, \infty)$.

Obviously, our $N=2$ model with "Big Bang" at $t=0$ reminds us of the elementary cosmological model of [23]. Once we accept such a cosmological interpretation of our present quantum evolution model, the finite interval of times $t \in(0,1)$ could be, in the same spirit, tentatively identified with the Inflation period, or with its initial quantized-evolution phase at least. The same terminological convention will be accepted to hold for the whole $N<\infty$ family of hiddenly Hermitian $N$ by $N$ matrices of $[13,18]$ involving Equation (3) as its most elementary special case. 


\subsection{Toy-Model Matrices with $N=3$}

The first nontrivial, three-by-three candidate for the crypto-Hermitian distance operator has the non-Hermitian real matrix form

$$
D^{(3)}(t)=\left[\begin{array}{ccc}
-2 & \sqrt{2} \tau(t) & 0 \\
-\sqrt{2} \tau(t) & 0 & \sqrt{2} \tau(t) \\
0 & -\sqrt{2} \tau(t) & 2
\end{array}\right]
$$

with $\tau(t)=\sqrt{1-t^{2}}$, with the real spectrum $\left\{d_{n}^{(3)}(t)\right\}=\{-2 t, 0,2 t\}$ and with the triple EP value $t_{(E P)}=0$.

The construction of the corresponding general two-parametric Hermitizing metrics $\Theta_{[\alpha, \beta]}(t)$ (we skip its presentation) as well as of its specific and unique product form $\Theta_{[0,0]}(t)=\mathcal{P} \mathcal{C}(t)$ remains routine. In terms of antidigonal unit matrix $J$ with Kronecker-delta elements $J_{i j}=\delta_{i+j, N+1}$, $i, j=1,2, \ldots, N$ we get the following result.

Lemma 1. For the crypto-Hermitian distance operator of the $N=3$ form (11) at times $t \in(0,1)$ the requirement $\Theta(t)=\mathcal{P C}(t)$ makes the Hermitizing HSM unique,

$$
\Theta_{[0,0]}^{(3)}(t)=I-\tau(t)\left[\begin{array}{ccc}
0 & \sqrt{2} & 0 \\
\sqrt{2} & 0 & \sqrt{2} \\
0 & \sqrt{2} & 0
\end{array}\right]+\tau^{2}(t) J
$$

Although one of the eigenvalues loses its positivity beyond the Big Bang boundary, i.e., at $\tau>\tau_{B B}=1$, the effect is spurious because it occurs at unphysical, purely imaginary times $t$. The behavior of the eigenvalues near $t=1$ alias $\tau(t)=0$ is more important because it shows that the end-of-Inflation limit of the conventional Dirac's metric $\Theta_{[0,0]}^{(3)}(1)=I$ is reached in a finite time after the Big Bang.

What remains to be proved or disproved is the minimal-anisotropy property of the physical and unique Hilbert space $\mathcal{H}^{(S)}(t)$ with metric (12). In order to shorten the analysis let us skip the detailed description of the fully general case and let us merely consider the following one-parametric subset of all of the solutions of Equation (7),

$$
\Theta^{(3)}(g, t)=\left[\begin{array}{ccc}
1 & -\sqrt{2} g \tau(t) & g \tau^{2}(t) \\
-\sqrt{2} g \tau(t) & 2 g-1+g \tau^{2}(t) & -\sqrt{2} g \tau(t) \\
g \tau^{2}(t) & -\sqrt{2} g \tau(t) & 1
\end{array}\right]
$$

For all of these HSM candidates guaranteeing the Hermiticity of operator $D^{(3)}(t)$ in $\mathcal{H}^{(S)}(t)$ we may evaluate the eigenvalues,

$$
\theta_{1,3}(g, t)=g \tau^{2}(t)+g \mp \sqrt{4 g^{2} \tau^{2}(t)+g^{2}-2 g+1}, \quad \theta_{2}(t)=1-g \tau^{2}(t)
$$

and demonstrate that the choice of the free parameter $g$ which would guarantee the minimal anisotropy of the geometry of Hilbert space $\mathcal{H}^{(S)}(t)$ is unique and equal to $g=1$. Such a result may be given the form of the following two lemmas.

Lemma 2. Whenever $g \neq 1$ there exists a real $\tau_{(\text {delayed })}<\tau_{B B}=1$ such that the HSM candidate (13) is positive definite if and only if $|\tau(t)|<\tau_{(\text {delayed })}$.

Proof. The use of ansatz $g=1+\epsilon$ in Equation (14) (with, say, $|\epsilon|<1$ ) converts the inequality $\theta_{1}<0$ into relation

$$
4\left(1-t^{2} / 2\right)^{2}<4\left(1-t^{2}\right)+\epsilon^{2} /(1+\epsilon)^{2}
$$


i.e.,

$$
t^{2}<\left|\frac{\epsilon}{1+\epsilon}\right|
$$

Thus, at each choice of $\epsilon \neq 0$ the inequality will hold in a non-empty interval of small $t$.

Lemma 3. Whenever $g \neq 1$, metric (13) is not isotropic at the "end-of-Inflation" time $t=1$.

Proof. follows from an immediate inspection of formula (13).

The single requirement of minimal anisotropy makes the metric optimal at $t=0$ and $t=1$, i.e., at both of the ends of the Inflation-resembling interval. The deviations of the roots (14) from their minimal-anisotropy extreme at $g_{\text {optimal }}=1$ are easily described. The middle root (forming an inverted parabola in $\tau$ ) remains positive for $g<1 / \tau^{2}$. The change of the sign of the smaller $\theta_{1}$ (which is positive for all $\tau^{2} \neq 1$ at $g=1$ ) may take place at the curves $g=1 / \tau^{2}$ (= the shared upper boundary of the domain of positivity for $\tau \in(0,1)$ ) and $g=1 /\left(2-\tau^{2}\right)$ (= the lower boundary) in the $g-\tau$ plane. Whenever $g \neq 1$, the Inflation-resembling evolution has no natural end because the time-dependent Hilbert-space geometry remains anisotropic at $t=1$ alias $\tau=0$.

\subsection{Toy-Model Matrices with $N=4$}

The latter two Lemmas offer a hint for an extrapolation of the pattern to the larger Hilbert-space dimensions $N>3$. With the next operator

$$
D^{(4)}(t)=\left[\begin{array}{cccc}
-3 & \sqrt{3} \tau(t) & 0 & 0 \\
-\sqrt{3} \tau(t) & -1 & 2 \tau(t) & 0 \\
0 & -2 \tau(t) & 1 & \sqrt{3} \tau(t) \\
0 & 0 & -\sqrt{3} \tau(t) & 3
\end{array}\right]
$$

(with the real spectrum $\left\{d_{n}^{(4)}(t)\right\}=\{-3 t,-t, t, 3 t\}$ and with the quadruple EP value $t_{(E P)}=0$ ), we get beyond the scope of the general analytic metric-operator results of [19]. Fortunately, even our $N=4$ example still admits a non-numerical treatment.

Lemma 4. Manifestly time-dependent matrix (15) is Hermitian with respect to the manifestly time-dependent HSM matrix

$$
\Theta^{(4)}(t)=\left[\begin{array}{cccc}
1 & -\sqrt{3} \tau(t) & \sqrt{3} \tau^{2}(t) & -\tau^{3}(t) \\
-\sqrt{3} \tau(t) & 1+2 \tau^{2}(t) & -2 \tau(t)-\tau^{3}(t) & \sqrt{3} \tau^{2}(t) \\
\sqrt{3} \tau^{2}(t) & -2 \tau(t)-\tau^{3}(t) & 1+2 \tau^{2}(t) & -\sqrt{3} \tau(t) \\
-\tau^{3}(t) & \sqrt{3} \tau^{2}(t) & -\sqrt{3} \tau(t) & 1
\end{array}\right]
$$

The eigenvalues $\left\{\theta_{1}, \ldots, \theta_{4}\right\}$ of the latter matrix form the ordered set

$$
\left\{1-3 \tau+3 \tau^{2}-\tau^{3}, 1-\tau-\tau^{2}+\tau^{3}, 1+\tau-\tau^{2}-\tau^{3}, 1+3 \tau+3 \tau^{2}+\tau^{3}\right\}
$$

and satisfy the positivity requirements during all Inflation, i.e., at all times $t \in(0,1)$.

Proof. The verification of the hidden Hermiticity (7) of operator (15) was performed using the routine, computer-assisted linear algebra. At $N=4$ these manipulations are still feasible and provide, as a byproduct, the closed-form eigenvalues of the physical Hilbert-space metric.

Figure 2 offers the graphical illustration of the latter observation. The time-variation of the eigenvalues is shown to start from the absence of any isotropy of Hilbert space $\mathcal{H}^{(S)}(t)$ at Big Bang instant $t=0$. Immediately after the Big Bang the degenerate $(N-1)$-plet of the vanishing eigenvalues of the metric is seen to unfold in a hierarchical, ordered manner. The smooth increase 
of the isotropy during Inflation ends, at $t=1$, by the ultimate disappearance of the anisotropy. This marks the end of the Inflation at finite time.

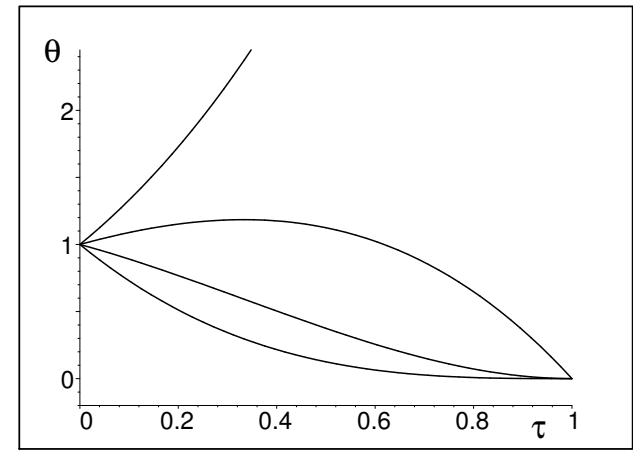

Figure 2. The quadruplet of eigenvalues (17) of the minimally anisotropic $N=4$ metric (16). The uppermost curve moves up and reaches its maximum $\theta_{\max }=8$ in the Big Bang limit of $\tau\left(t_{B B}\right)=1$.

\section{Discussion}

It is important to keep in mind that our present matrix models still represent just a very schematic realization of the underlying idea of the quantization of the measurable distance between Alice and Bob. Naturally, in the context of a not yet existing full fledged quantum cosmology we are still far from any truly realistic picture. At the same time it is quite plausible that that the basic features our elementary toy models may be rather realistic even under our drastic simplifications.

First of all we believe that our present purely pedagogical restriction of attention to the smallest dimensions $N$ of the spatial grid is not too relevant and that the underlying background-independent quantum dynamics of the expanding Universe at short times will remain qualitatively unchanged at all of the finite matrix dimensions $N$.

It is possible to claim that on the purely mathematical side our present results clearly support the idea of the feasibility of quantization in which several traditional "no-go" expectations are circumvented via the identification of the spatiotemporal coordinates of quantum catastrophes like Big Bang with the Kato's exceptional-point parameters of the theory.

On the purely formal level we connected here the implementation of this idea with an active use of the manifestly time-dependent forms of quantum theory in which the observables exhibit a hidden Hermiticity, i.e., in which the (perhaps, necessarily needed) new physical degrees of freedom emerge as rrepresented by the Hilbert-space inner-product metric $\Theta$. Then, in a way paralleling the phenomenological quantum-catastrophe perspective of [20] the initial choice of the cryptohermitian operators of observables (i.e., in our simplified models, of the "quantized distance" matrices $D^{(N)}(t)$ ) represents one of the most efficient strategies in model-building.

Via a detailed analysis of our models we showed that even when any form of the dynamical input information is allowed to be manifestly time-dependent, the technical manipulations (and, first of all, the constructions of physical metric) remain - at least conceptually - feasible. Thus, in methodical sense we may be quite assertive, opposing, e.g., the scepticism [6,46-48], and joining opinions [49,50], the authors of which managed to clarify several comparatively widespread though just more or less purely terminological misunderstandings.

Naturally, also our present constructions combine the clarification of the innovative methodology with a concrete sample of its possible immediate impact upon the predictions to be made by any realistic theory in quantum cosmology. In such an applied-theory context we are persuaded that especially the present, virtually serendipitous discovery of possible connection between the process of quantization and the emergence of the Inflation-resembling short time-period after the Big Bang sounds quite inspiring. 
The generalization of the formalism in which the coupling $\lambda$ in Hamiltonians $H(\lambda)$ would vary with time appeared to require a thorough change of the formalism [24,40]. Moreover, it also proved accompanied by a perceivable loss of its simplicity [34,51-55]. Fortunately, the major part of these discouraging shortcomings disappears after one disconnects the toy-model matrices from their early role of Hamiltonians (i.e., roughly speaking, of the energy operators). Such a change of interpretation formed in fact the core of our present message. Marginally, let us add that the methodical relevance of the time-dependent observables (sampled by (3), (11) and (15), etc.) in quantum cosmology was preliminarily communicated also in a recent conference [56]. The videorecorded form [57] of the talk on the webpage of the conference might be also recalled as a predecessor and/or complement of the present text.

\section{Summary}

Via toy-model examples we managed here to demonstrate that the spatial Big Bang singularity of classical cosmology need not necessarily be replaced by a regularized Big Bounce after quantization. Our demonstration proceeded, constructively, via a non-covariant, highly schematic model of an empty one-dimensional Universe in which a pair of experimentalists (Alice and Bob) measure their distance.

As an unpredicted byproduct of the study of toy models we revealed that in their cosmological phenomenological implementations the Big Bang appears followed by a finite anomalous evolution period carrying multiple features of a hypothetical "Inflation". Thus, the period of Inflation appears naturally identified with the period of an anisotropy of the "physical" Hilbert space of the hypothetical (i.e., "multiverse") quantum states of the non-stationary, evolving Universe. In other words, during the evolutionary stage which mimics (explains?) Inflation one encounters a step-by-step weakening of the anisotropy of the Hilbert space of states of the Universe.

Thus, with the Big Bang singularity identified with the Kato's exceptional point, the Inflation period is explained as a purely quantum phenomenon. In this sense our present results may be directly related to our older quantum-cosmology-related conjecture [34] where we already tried to work, constructively, with a doublet of the non-Hermitian operators of observables. Unfortunately, the latter conjecture was based on an inefficient strategy as long as we kept the Hamiltonian $H \neq H^{\dagger}$ maximally elementary (i.e., constant and parameter-independent) while having started our constructions from the time-dependent set of eligible Hermitizing metrics $\Theta(t)$. This made the older construction of the present spatial-geometry observable $D(t)$ (which would characterize the Big Bang or Big Crunch in the respective limits $t \rightarrow t_{\text {critical }}$ ) prohibitively complicated. Due to the emergent technical complications we only managed to construct the relevant resulting $D(t)=D^{(N)}(t)$ at $N=2$.

In the present paper we made use of a much more efficient strategy. It was based, in essence, just on a reversal of the flow of the construction. Thus, we started now directly from a suitable initial choice of the time-dependent candidates $D^{(N)}(t)$ ) for the "geometry" observable (viz., the Alice-Bob distance). Having made use of the discretization philosophy as advocated and tested in [58], manifestly non-Hermitian matrices $D^{(N)}(t)$ were chosen also here, playing the role of the operators of an observable distance. Thus, the time-dependent eigenvalues of $D^{(N)}(t)$ were assumed to be measured and given the cosmological toy-model geometrical meaning.

Acknowledgments: The work on the project was supported by the Institutional Research Plan RVO61389005 and by GAČR Grant Nr. 16-22945S.

Conflicts of Interest: The authors declare no conflict of interest.

\section{References}

1. Bender, C.M.; Boettcher, S. Real spectra in non-Hermitian Hamiltonians having PT symmetry. Phys. Rev. Lett. 1998, 80, 5243. 
2. Bender, C.M.; Milton, K.A. Nonperturbative calculation of symmetry breaking in quantum field theory. Phys. Rev. D 1997, 55, 3255-3259.

3. Albeverio, S.; Kuzhel, S. PT-symmetric operators in quantum mechanics: Krein spaces methods. In Non-Selfadjoint Operators in Quantum Physics: Mathematical Aspects; John Wiley \& Sons: Hoboken, NJ, USA, 2015; pp. 293-344.

4. Caliceti, E.; Graffi, S. Criteria for the reality of the spectrum of PT-symmetric Schrödinger operators and for the existence of PT-symmetric phase transitions. In Non-Selfadjoint Operators in Quantum Physics: Mathematical Aspects; John Wiley \& Sons: Hoboken, NJ, USA, 2015; pp. 189-240.

5. Bender, C.M. Making Sense of Non-Hermitian Hamiltonians. Rep. Prog. Phys. 2007, 70, 947-1018.

6. Mostafazadeh, A. Pseudo Hermitian representation of quantum mechanics. Int. J. Geom. Meth. Mod. Phys. 2010, 7, 1191-1306.

7. Znojil, M. Non-selfadjoint operators in quantum physics: Ideas, people and trends. In Non-Selfadjoint Operators in Quantum Physics: Mathematical Aspects; John Wiley \& Sons: Hoboken, NJ, USA, 2015; pp. 7-58.

8. Bagarello, F.; Gazeau, J.-P.; Szafraniec, F.H.; Znojil, M. (Eds.) Non-Selfadjoint Operators in Quantum Physics: Mathematical Aspects; John Wiley \& Sons: Hoboken, NJ, USA, 2015.

9. PT Symmetry-the Workshop's Homepage. Available online: http://gemma.ujf.cas.cz/ znojil/conf/ (accessed on 16 May 2016).

10. Stone, M.H. On one-parameter unitary groups in Hilbert Space. Ann. Math. 1932, 33, 643-648.

11. Kato, T. Perturbation Theory for Linear Operators; Springer-Verlag: Berlin, Germnay, 1966.

12. Siegl, P.; Krejcirik, D. On the metric operator for the imaginary cubic oscillator. Phys. Rev. D 2012, $86,121702(\mathrm{R})$.

13. Znojil, M. Maximal couplings in PT-symmetric chain-models with the real spectrum of energies. J. Phys. A Math. Theor. 2007, 40, 4863-4875.

14. Znojil, M. Matrix Hamiltonians with a chance of being complex symmetric. Integral Equ. Oper. Theory 2012, 74, 5-6.

15. Dyson, F.J. General Theory of Spin-Wave Interactions. Phys. Rev. 1956, 102, 1217-1230.

16. Dieudonné, J. Quasi-Hermitian Operators. Proc. Int. Symp. Lin. Spaces; Pergamon: Oxford, UK, 1961; pp. 115-122.

17. Scholtz, F.G.; Geyer, H.B.; Hahne, F.J.W. Quasi-Hermitian operators in Quantum Mechanics and the variational principle. Ann. Phys. 1992, 213, 74-101.

18. Znojil, M. Tridiagonal PT-symmetric $N$ by $N$ Hamiltonians and a fine-tuning of their observability domains in the strongly non-Hermitian regime. J. Phys. A Math. Theor. 2007, 40, 13131-13148.

19. Znojil, M. Horizons of stability. J. Phys. A Math. Theor. 2008, 41, 44027.

20. Znojil, M. Quantum catastrophes: A case study. J. Phys. A Math. Theor. 2012, 45, 444036.

21. Zeeman, F.C. Catastrophe Theory-Selected Papers 1972-1977; Addison-Wesley: Reading, MA, USA, 1977.

22. Arnold, V.I. Catastrophe Theory; Springer: Berlin, Germany, 1984.

23. Borisov, D.I.; Ruzicka, F.; Znojil, M. Multiply Degenerate Exceptional Points and Quantum Phase Transitions. Int. J. Theor. Phys. 2015, 54, 4293-4305.

24. Znojil, M. Three-Hilbert-space formulation of Quantum Mechanics. SIGMA 2009, 5, 001.

25. Znojil, M. On the role of the normalization factors $\kappa_{n}$ and of the pseudo-metric P in crypto-Hermitian quantum models. SIGMA 2008, 4, 001.

26. Mostafazadeh, A.; Batal, A. Physical Aspects of Pseudo-Hermitian and PT-Symmetric Quantum Mechanics. J. Phys. A Math. Theor. 2004, 37, 11645-11679.

27. Turbiner, A. Personal Communication, Mexico City, Mexico, 27 April 2000.

28. Borisov, D.I.; Znojil, M. Mathematical and physical meaning of the crossings of energy levels in PT-symmetric systems. In Non-Hermitian Hamiltonians in Quantum Physics; Bagarello, F., Ed.; Springer: Basel, Switzerland, 2016.

29. El-Ganainy, R.; Makris, K.G.; Christodoulides, D.N.; Musslimani, Z.H. Theory of coupled optical PT-symmetric structures. Opt. Lett. 2007, 32, 2632-2634.

30. Makris, K.G.; El-Ganainy, R.; Christodoulides, D.N.; Musslimani, Z.H. Optical Solitons in $\mathcal{P} \mathcal{T}$ Periodic Potentials. Phys. Rev. Lett. 2008, 100, 030402.

31. Makris, K.G.; El-Ganainy, R.; Christodoulides, D.N.; Musslimani, Z.H. Beam dynamics in $\mathcal{P} \mathcal{T}$ symmetric optical lattices. Phys. Rev. Lett. 2008, 100, 103904. 
32. Znojil, M. Scattering theory using smeared non-Hermitian potentials. Phys. Rev. D. 2009, 80, 045009.

33. Musslimani, Z.H.; Makris, K.G.; El-Ganainy, R.; Christodoulides, D.N. J. Phys. A Math. Theor. 2008, 41, 244019.

34. Znojil, M. Quantum Big Bang without fine-tuning in a toy-model. J. Phys. Conf. Ser. 2012, 343, 012136.

35. Ashtekar, A.; Pawlowski, T.; Singh, P. Quantum nature of the big bang: Improved dynamics. Phys. Rev. D 2006, 74, 084003.

36. Ashtekar, A.; Corichi, A.; Singh, P. Robustness of key features of loop quantum cosmology. Phys. Rev. D 2008, 77, 024046.

37. Znojil, M. Non-Hermitian Heisenberg representation. Phys. Lett. A 2015, 379, 2013-2017.

38. Malkiewicz, P.; Piechocki, W. Turning Big Bang into Big Bounce: II. Quantum dynamics. Class. Quant. Gravity 2010, 27, 225018.

39. Bergeron, H.; Czuchry, E.; Gazeau, J.-P.; Malkiewicz, P.; Piechocki, W. Singularity avoidance in a quantum model of the Mixmaster universe. Phys. Rev. D 2015, 92, 124018.

40. Znojil, M. Time-dependent version of cryptohermitian quantum theory. Phys. Rev. D 2008, 78, 085003.

41. Mukhanov, V. Physical Foundations of Cosmology; Cambridge University Press: Cambridge, UK, 2005.

42. Rovelli, C. Quantum Gravity; Cambridge University Press: Cambridge, UK, 2004.

43. Znojil, M.; Geyer, H.B. Construction of a unique metric in quasi-Hermitian quantum mechanics: Nonexistence of the charge operator in a $2 \times 2$ matrix model. Phys. Lett. B 2006, 640, 52-56.

44. Znojil, M.; Geyer, H.B. Erratum to: "Construction of a unique metric in quasi-Hermitian quantum mechanics: Nonexistence of the charge operator in a $2 \times 2$ matrix model" [Phys. Lett. B 640 (2006) 52]. Phys. Lett. B 2007, 649, 494.

45. Bender, C.M.; Brody, D.C.; Jones, H.F. Complex Extension of Quantum Mechanics. Phys. Rev. Lett. 2002, 89,270401

46. Streater, R.F. Lost Causes in and Beyond Physics; Springer: Berlin, Germany, 2007.

47. Lee, Y.-C.; Hsieh, M.-H.; Flammia, S.T.; Lee, R.-K. Local PT symmetry violates the no-signalling principle. Phys. Rev. Lett. 2014, 112, 130404;

48. Croke, S. PT-symmetric Hamiltonians and their application in quantum information. Phys. Rev. A 2015, 91, 052113.

49. Brody, D.C. Consistency of PT-symmetric quantum mechanics. J. Phys. A Math. Theor. 2016, 49, 10 LT03.

50. Fring, A.; Moussa, M.H.Y. Unitary quantum evolution for time-dependent quasi-Hermitian systems with non-observable Hamiltonians. Phys. Rev. A 2016, 93, 042114.

51. De Morisson, C.F.; Fring, A. Time evolution of non-Hermitian Hamiltonian systems. J. Phys. A Math. Gen. 2006, 39, 9269-9289.

52. Bíla, H. Non-Hermitian Operators in Quantum Physics. Ph.D. Thesis, Charles University, Prague, Czech Republic, 2008

53. Gong, J.-B.; Wang, Q.-H. Geometric phase in PT-symmetric quantum mechanics. Phys. Rev. A 2010, 82, 012103.

54. Znojil, M. Crypto-unitary forms of quantum evolution operators. Int. J. Theor. Phys. 2013, 52, $2038-2045$.

55. Maamache, M. Periodic pseudo-Hermitian Hamiltonian: Nonadiabatic geometric phase. Phys. Rev. A 2015, 92, 032106 .

56. Quantum (and Classical) Physics with Non-Hermitian Operators (PHHQP13). Available online: http:/ / www.as.huji.ac.il/isf/phhqp13 (accessed on 14 June 2016).

57. Recorded Lectures-PHHQP13-The Institute for Advanced Studies. Available online: http://www.as.huji. ac.il/node/877 (accessed on 14 June 2016).

58. Znojil, M. The cryptohermitian smeared-coordinate representation of wave functions. Phys. Lett. A 2011, 375, 3176-3183.

(C) 2016 by the author; licensee MDPI, Basel, Switzerland. This article is an open access article distributed under the terms and conditions of the Creative Commons Attribution (CC-BY) license (http://creativecommons.org/licenses/by/4.0/). 